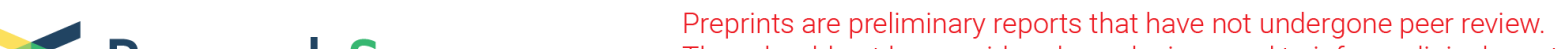 Research Square
They should not be onsidideded conllusive, used to inform clinical practice,
or referenced by the media salidated information.
}

\section{High uptake of 68Ga-PSMA-HBED-CC and 18F- DCFPyL in the peritumoral area of rat gliomas due to activated astrocytes}

\section{Dennis Oliveira}

Forschungszentrum Julich Institut fur Neurowissenschaften und Medizin

\section{Carina Stegmayr}

Forschungszentrum Julich Institut fur Neurowissenschaften und Medizin

\section{Alexander Heinzel}

Universitatsklinikum Aachen

\section{Johannes Ermert}

Forschungszentrum Julich Institut fur Neurowissenschaften und Medizin

\section{Bernd Neumaier}

Forschungszentrum Julich $\mathrm{GmbH}$

\section{Nadim Jon Shah}

Forschungszentrum Julich Institut fur Neurowissenschaften und Medizin

\section{Felix M. Mottaghy}

Universitatsklinikum Aachen

Karl-Josef Langen ( $\boldsymbol{\nabla}$ k.j.langen@fz-juelich.de )

Forschungszentrum Jülich https://orcid.org/0000-0003-1101-5075

\section{Antje Willuweit}

Forschungszentrum Julich Institut fur Neurowissenschaften und Medizin

\section{Original research}

Keywords: 68Ga-PSMA, 18F-DCFPyL, prostate specific antigen, rat gliomas, reactive astrocytosis

Posted Date: March 17th, 2020

DOI: https://doi.org/10.21203/rs.3.rs-17419/v1

License: (c) (i) This work is licensed under a Creative Commons Attribution 4.0 International License. Read Full License

Version of Record: A version of this preprint was published at EJNMMI Research on May 25th, 2020. See the published version at https://doi.org/10.1186/s13550-020-00642-0. 


\section{Abstract}

Background Recent studies reported on high uptake of the PSMA ligands [68Ga]HBED-CC (68Ga-PSMA) and 18F-DCFPyL in cerebral gliomas. This study explores the regional uptake and cellular targets of 68Ga-PSMA and 18F-DCFPyL in three different rat glioma models.

Methods $\mathrm{F98}$, 9L or U87 rat gliomas were implanted into the brains of 38 rats. After 13 days of tumor growth, 68Ga-PSMA $(n=21)$ or 18F-DCFPyL $(n=17)$ were injected intravenously and animals were sacrificed 40 min later. Cryosections of the tumor bearing brains were analyzed by ex vivo autoradiography and immunofluorescence staining for blood vessels, microglia, astrocytes and presence of PSMA. Blood-brain barrier (BBB) permeability was tested by coinjection of Evans blue dye (EBD). 68Ga-PSMA uptake after restoration of BBB integrity by treatment with dexamethasone (Dex) was evaluated in four animals with U87 gliomas. Competition experiments using the PSMA-receptor inhibitor 2-(Phosphonomethyl)pentane-1,5-dioic acid (PMPA) were performed for both tracers in two animals each.

Results All tumors showed a strong 68Ga-PSMA and 18F-DCFPyL binding in the peritumoral area and moderate binding in the center of the tumors. PMPA administration led to complete inhibition of $68 \mathrm{Ga}$ PSMA and 18F-DCFPyL binding in the peritumoral region. Restoration of BBB by Dex treatment reduced EBD extravasation but $68 \mathrm{Ga}$-PSMA binding remained unchanged. Expression of activated microglia (CD11b) was low in the intra- and peritumoral area but GFAP staining revealed strong activation of astrocytes in congruency to the tracer binding in the peritumoral area.

Conclusions High uptake of 68Ga-PSMA and 18F-DCFPyL in the peritumoral area of all glioma models is presumably caused by activated astrocytes. This may represent a limitation for the clinical application of PSMA ligands in gliomas. BBB permeability had a minor influence on tracer binding.

\section{Introduction}

PET ligands for the prostate specific membrane antigen (PSMA) are very successful in the diagnostic assessment of prostate cancer $[1,2]$. The most widely studied agent is the ${ }^{68} \mathrm{Ga}$-labelled PSMA inhibitor Glu-NH-CO-NH-Lys(Ahx)-HBED-CC ( $\left.{ }^{68} \mathrm{Ga}-\mathrm{PSMA}\right)$ but in recent years ${ }^{18} \mathrm{~F}$-labelled ligands such as 2-(3-(1carboxy-5-[(6-[ $\left.{ }^{18} \mathrm{~F}\right]$ fluoro-pyridine-3-carbonyl)-amino]-pentyl)-ureido)-pentanedioic acid ( ${ }^{18} \mathrm{~F}$-DCFPyL) have been developed which show advantages with respect to production amount, availability, clinical utility, and image resolution [3, 4]. PSMA is also expressed by a variety of non-prostate cancers, often on the endothelium of tumor-associated neovasculature [5] and initial studies have explored the application of PSMA ligands in breast, lung, bladder, pancreatic and colorectal cancer, renal cell carcinoma and glioblastoma [6]. In a triple negative breast cancer xenograft the localization of PSMA was detected in the xenograft-associated endothelial cells as well as on the tumor cells [7]. Also benign tissue like the cervical ganglia possess a very strong PSMA expression [8].

Most of the reported findings with PSMA-targeted radiotracers in non-prostate malignancies, however, are limited to small series of patients and further investigations are needed to explore the potential of PSMA 
ligands outside prostate cancer.

Glioblastoma is the most frequent malignant adult brain tumor and has a dismal prognosis. Various approaches have focused on the application of radionuclides for targeted therapy of these tumors [9]. Previous histopathological studies of human glioblastoma have identified PSMA on neovasculature of gliomas as a potential target for treatment $[10,11]$. Subsequent pilot studies have reported on high uptake of ${ }^{68} \mathrm{Ga}$-PSMA and ${ }^{18} \mathrm{~F}$-DCFPyL in brain tumors suggesting a potential role of PSMA ligands in theranostics [12-15]. It remains unclear, however, whether the binding of the PSMA ligands occurs mainly on tumor cells or endothelial cells and to what extent the accumulation is influenced by other factors such as disturbance of the blood-brain barrier (BBB). Furthermore, it is unknown to what extent the ligands bind to activated microglia and astroglia which are the two major types of glial cells involved in the regulation of the immune response to pathological processes in the brain and which could affect the specificity of the tracers for tumor detection.

In this study, we explored the regional binding of the well-established PSMA ligands ${ }^{68} \mathrm{Ga}-\mathrm{PSMA}$ and ${ }^{18} \mathrm{~F}$ DCFPyL in three different rat glioma models including the human U87 glioma cell line. Special attention was paid to the evaluation of the role of microglia and astroglia, the role of BBB permeability, which was modulated by treatment with dexamethasone (Dex), and the specificity of tracer binding which was assessed by competition experiments with the PSMA-receptor inhibitor PMPA. The experiments provided interesting new insight into the cellular targets that might be involved in the accumulation of PSMA ligands in cerebral gliomas.

\section{Material And Methods}

\section{Animals}

Thirty-eight male rats (Charles River Laboratories, Sulzfeld, Germany) were included in this study. An overview of the experiments, the rat strains, glioma models, tracers, pharmacological interventions and number of animals in each group is given in Table 1. All animals were handled in accordance with the Animal Research Committee of the Forschungszentrum Jülich $\mathrm{GmbH}$, the German Animal Welfare Act and the European Community Council directives regarding the protection of animals used for experimental and scientific purposes (2010/63/EU), and with approval by the local ethics committee (Landesamt für Natur, Umwelt und Verbraucherschutz, North-Rhine-Westphalia, Germany, Az: 8402.04.2016.248). All rats weighted between 230 and $330 \mathrm{~g}$ and were housed in groups of two under standard conditions. Food and water was provided ad libitum.

\section{Cell culture and tumor inoculation}

U87 and 9L tumor cells (U87: ATCC $\AA$ HTB-14 ${ }^{\mathrm{TM}}$, LGC Standards GmbH; 9L: ECACC GS-9L, Salisbury, UK) were cultured in Minimum Essential Medium Eagle (MEM) supplemented with 10\% fetal calf serum, $1 \%$ 
glutamine, $1 \%$ penicillin/streptomycin and 1\% non-essential amino acids. F98 tumor cells were cultured similar to U87 and 9L, but in Dulbecco's Modified Eagle's Medium (DMEM) instead of MEM and without non-essential amino acids. After reaching about $95 \%$ of confluency, cells were prepared for inoculation into rat brains. Therefore, cells were washed with PBS and detached by incubation with a trypsin/EDTA solution for $5 \mathrm{~min}$. Afterwards cells were resuspended in cell medium generating a cell concentration of 500.000 cells $/ 5 \mu \mathrm{l}$ for the U87 cells, $65.000 / 5 \mu \mathrm{l}$ for the $9 \mathrm{~L}$ cells and $30.000 / 5 \mu \mathrm{l}$ for the F98 cells.

Cell suspensions were then stereotactically inoculated into the left anterior striatum under anesthesia as described previously [16]. The tumor was allowed to grow for 13 days.

\section{Autoradiography}

${ }^{18} \mathrm{~F}$-DCFPyL and ${ }^{68} \mathrm{Ga}$-PSMA were synthesized as described previously [17]. On day 13 post surgery, ex vivo autoradiography (AR) was performed on 38 rats 40 min after i.v. injection of approximately $40 \mathrm{MBq}$ ${ }^{68} \mathrm{Ga}$-PSMA or ${ }^{18} \mathrm{~F}$-DCFPyL, respectively (Table 1). For the AR, animals were sacrificed, rat brains removed and immediately frozen in liquid isopentane $(-50 \mathrm{C})$. Every tenth slice of the $20 \mu \mathrm{m}$ cryosections of the tumor bearing brain and freshly prepared $20 \mu \mathrm{m}^{68} \mathrm{Ga}$ and ${ }^{18} \mathrm{~F}$ standards, to generate a tracer calibration curve, were exposed to an imaging plate (Fuji Imaging Plate, Raytest) overnight, scanned (Fuji BAS Reader 5000, Raytest) and quantitatively evaluated with a pixel size of $25 \mu \mathrm{m}$ (AIDA Version 4.50, Raytest). Tracer uptake in the tissue was expressed as standardized uptake value (SUV) by dividing the radioactivity $(\mathrm{kBq} / \mathrm{ml})$ in the tissue by the radioactivity injected per gram of body weight.

\section{Histological staining}

Cryosections of the brains were stained with DAPI (4',6-diamidino-2-phenylindole) and evaluated by fluorescence microscopy. Disturbance of the BBB was visually evaluated using Evans blue dye (EBD). Therefore, $500 \mu \mathrm{l} / \mathrm{kg} 2 \%$ EBD was injected i.v. $30 \mathrm{~min}$ before decapitation of the rat for AR. EBD extravasation in brain slices was examined by fluorescence microscopy (LMD6000, Leica Microsystems CMS GmbH) and processed by AIDA software (AIDA Version 4.50, Raytest).

Blood vessels were stained with anti-rat von Willebrand factor antibody (ab6994, Abcam). To visualize activated microglia, anti-rat CD11b (Integrin alpha M) antibody (ab133357, Abcam) was used. Reactive astrocytes were visualized by staining with anti-rat GFAP (glial fibrillary acidic protein) antibody (ab53554, Abcam). Immunofluorescence PSMA staining was applied to tumor bearing brain slices using three different anti-PSMA antibodies (NBP1-45057 and NBP1-89822, Novus Biologicals; ab58779, Abcam) to display PSMA expression. To verify the functionality and specificity of the used PSMA antibodies, rat prostate and kidney tissue were used as positive controls. Immunofluorescence stainings were performed according to standard histology protocols and as described before [18]. 


\section{Pharmacological interventions}

Competition tests were performed with four animals to evaluate binding specificity of ${ }^{68} \mathrm{Ga}-\mathrm{PSMA}(\mathrm{n}=2)$ and ${ }^{18}$ F-DCFPyL ( $\mathrm{n}=2$ ). As competitor $30 \mathrm{mg} / \mathrm{kg}$ BW 2-(Phosphonomethyl)pentane-1,5-dioic acid (PMPA; Tocris) was co-injected i.v. with the tracer as described in the literature [19]. After 40 min of incubation autoradiography was performed as described above.

In order to evaluate the influence of BBB permeability on tracer uptake, four rats with U87 gliomas received glucocorticoid treatment with Dexamethasone (Jenapharm ${ }^{\circledR}, \mathrm{Mibe} \mathrm{GmbH}$ ). The rats received 8 $\mathrm{mg} / \mathrm{kg}$ intraperitoneally on day 11 post surgery and $4 \mathrm{mg} / \mathrm{kg}$ each on days 12 and 13 . Autoradiography was performed after injection of ${ }^{68} \mathrm{Ga}$-PSMA as described above.

\section{Data evaluation}

The autoradiograms were co-registered to the DAPI stained sections of adjacent slices to compare tracer binding to morphological data as described previously [18]. In short, a circumference region-of-interest (ROI) was drawn along the borders of the coronal brain slices in the DAPI stained sections. After adapting the size of the corresponding autoradiogram to that ROI, tracer binding in the tumor region was evaluated by three different ROIs: 1) along the outer tumor margin reflecting the total tumor volume, 2) in the central part of the tumor and 3) around the peritumoral area. The ROI in the center of the tumor reflects tracer binding in the tumor tissue, excluding the prominent spherical tracer binding in the peritumoral area. The $\mathrm{ROI}$ on the peritumoral area contained only the prominent spherical tracer binding, without the binding in the center. Furthermore, a reference ROI was placed in the normal brain tissue and tumor-to-background ratios (TBR) were calculated by dividing the mean SUV of the different tumor ROls by the mean SUV of the background ROI. Data evaluation included the tumor volume $\left(\mathrm{mm}^{3}\right)$, SUV in the ROIs and TBRs.

\section{Statistics}

Statistical evaluation was performed using Sigma Plot 12.5 (Systat Software GmbH). Two-Way repeated measures ANOVAs with Holm-Sidak post-hoc test were performed 1) to compare the volume of total tracer binding in the AR to the histological tumor volume derived from the nuclear staining, 2) to compare tracer uptake in the different tumor models for the AR data; and 3) for the comparison of control animals versus Dex treated animals. For analysis of astrocytes, comparison of staining intensity between the two treatment groups was performed by t-test.

\section{Results}

\section{Autoradiography}


Visual evaluation of the autoradiograms of the tumor bearing animals revealed a prominent spherical binding of both ${ }^{68} \mathrm{Ga}$-PSMA and ${ }^{18} \mathrm{~F}$-DCFPyL at the rim of the tumors and a lower binding within the center of the tumors in all three tumor models (Fig. 1, suppl. Fig 1). In contrast to the $9 \mathrm{~L}$ and U87 tumor model, all F98 tumors showed a central necrosis which showed slightly increased tracer binding. The coregistration of the autoradiographic and histological data demonstrated that the prominent spherical accumulation of the PSMA ligands projected on the peritumoral tissue outside the solid tumor mass. Correspondingly, total tumor volume in autoradiography was significantly larger than histological tumor volume for both tracers in all tumor models $(p<0.001)($ Tab. 2$)$.

SUV in the center of the tumors was significantly higher for ${ }^{68} \mathrm{Ga}$-PSMA than for ${ }^{18} \mathrm{~F}$-DCFPyL in all tumor models. Furthermore, the TBR of tracer uptake at the tumor rim was significantly higher for ${ }^{18} \mathrm{~F}$-DCFPyL than for ${ }^{68} \mathrm{Ga}$-PSMA in all tumor models (Tab. 3). Furthermore, high uptake was noted in the circumventricular organs, namely the organum vasculosum of the lamina terminalis (suppl. Fig 1), the median eminence, and the subfornical organ (data not shown).

\section{Pharmacological interventions}

Competition experiments using the PSMA specific ligand PMPA led to a disappearance of the increased spherical tracer binding in the peritumoral area (Fig. 2) and the TBR of ${ }^{68} \mathrm{Ga}$-PSMA and ${ }^{18} \mathrm{~F}$-DCFPyL uptake decreased considerably with PMPA (suppl. Tab. 1). Furthermore, tracer uptake in the circumventricular organs was completely surpressed by PMPA treatment (data not shown).

Dex treatment of U87 glioma bearing animals led to an almost complete reduction of EBD extravasation, indicating a restoration of the BBB or at least drastic reduction of BBB permeability (Fig. 3). In contrast,

${ }^{68} \mathrm{Ga}$-PSMA uptake showed no significant difference in the untreated and Dex treated U87 gliomas indicating that the change of BBB permeability had only a minor influence on tracer uptake in the tumors (Tab. 4).

\section{PSMA staining}

PSMA expression in the tumor and the peritumoral area was tested by three different PSMA antibodies. The antibody ab58779 demonstrated no staining in the peritumoral or the tumor center area while rat prostate and kidney tissue showed a positive staining (data not shown). The antibody NBP1-89822 showed positive staining of $9 \mathrm{~L}$ and F98 tumor cells (suppl. Fig. 2), but no staining in the peritumoral region. U87 tumors were negative (data not shown). The antibody NBP1-45057 showed a positive staining on vessel-like structures in all glioma models (Fig. 4; suppl. Fig. 2) but no staining in the peritumoral area.

\section{Staining with cellular markers}


The vasculature of the tumors was stained with the anti-von Willebrand factor antibody, which visualized dense and enlarged vessels inside the tumor and in the peritumoral area of all glioma models (Fig. 4; suppl. Fig. 2). There was no evidence of a higher density of blood vessels in the peritumoral area or at the tumor border in comparison to the main tumor mass.

CD11b staining demonstrated low presence of activated microglia in the tumor and/or the peritumoral area (Fig. 4; suppl. Fig. 2).

In contrast, GFAP staining of reactive astrocytes showed a strong signal in the peritumoral area in all tumor models in congruence with the prominent spherical binding of ${ }^{68} \mathrm{Ga}$-PSMA and ${ }^{18} \mathrm{~F}$-DCFPyL. Very few astrocytes were noted in the center of the tumors. An example of GFAP staining of U87 glioma is shown in Fig. 4, for 9L and F98 glioma in suppl. Fig. 2.

\section{Discussion}

In recent years, PET imaging using PSMA-selective tracers like ${ }^{68} \mathrm{Ga}-\mathrm{PSMA}$ has been shown to be a very sensitive and specific method for the diagnosis of prostate cancer, as PSMA is highly expressed in prostate carcinoma cells [20]. High PSMA expression, however, has also been shown in the neovasculature of solid tumors, including gliomas, making this a promising approach for brain tumor targeting $[10,11]$. Recent studies reported on high binding of ${ }^{68} \mathrm{Ga}$-PSMA and ${ }^{18} \mathrm{~F}$-DCFPyL in human brain tumors and presence of PSMA was immunohistochemically identified either in tumor cells or in neovasculature of these tumors [13-15]. However, direct binding of PSMA ligands to a specific cell population within the tumor tissue has not been proven so far. In addition, it remains unclear to what extent BBB permeability influences the uptake of PSMA ligands in gliomas. Therefore, we analyzed the ${ }^{68} \mathrm{Ga}$-PSMA and ${ }^{18} \mathrm{~F}$-DCFPyL distribution in different rat gliomas using autoradiography in comparison to various histological stainings. Furthermore, the specificity of tracer binding and the role of BBB permeability was addressed.

Surprisingly, autoradiography revealed a prominent spherical uptake of ${ }^{68} \mathrm{Ga}-\mathrm{PSMA}$ and ${ }^{18} \mathrm{~F}$-DCFPyL around the U87, $9 \mathrm{~L}$ and F98 gliomas, which was similar for both tracers (Fig. 1, suppl. Fig 1). Comparison with histological staining revealed that the tracer binding clearly projected outside the solid tumor mass in the peritumoral region. Previous PET studies in glioma patients did not report such findings, which may be explained by the considerably lower spatial resolution of PET compared with the ex vivo autoradiography used here.

Tracer binding in the tumors was further analyzed by competition experiments using the PSMA inhibitor PMPA [21]. PMPA is highly specific to PSMA, which has been demonstrated by lack of binding to more than 100 different receptors, ion channels, transporters and enzymes [22]. PMPA as well as the PSMAselective tracers bind to the active site on the extracellular part of the transmembrane protein PSMA. The co-injection of PMPA led to a complete suppression of ${ }^{68} \mathrm{Ga}$-PSMA and ${ }^{18} \mathrm{~F}$-DCFPyL binding in the peritumoral areas of $\mathrm{U} 87$ and $9 \mathrm{~L}$ glioma, respectively (Fig. 2) and to a reduction of tracer uptake in the 
center of the tumors. These findings strongly suggest that the observed phenomenon of tracer uptake in the periphery of the tumors reflects specific binding to PSMA receptors.

The influence of BBB permeability on tracer uptake was investigated by treatment with the glucocorticoid Dex as described previously [23]. A partial restoration of BBB of the tumors by Dex treatment led to a considerable decrease of Evans blue extravasation into the tumor tissue of Dex treated rats. In contrast, no significant differences of ${ }^{68} \mathrm{Ga}$-PSMA uptake between untreated and Dex treated U87 gliomas were observed, especially at the rim of the tumors suggesting that the influence of BBB permeability on tracer binding, especially in the periphery of the tumors is small.

Immunofluorescence staining using three different anti-PSMA antibodies showed signals on tumor cells in 9L and F98 gliomas and on vessel-like structures in U87, 9L and F98 gliomas (Fig. 4, suppl. Fig 2) similar to observations in human gliomas $[10,11]$. However, no PSMA staining was observed in the peritumoral region. One possible explanation for this finding is the existence of different PSMA isoforms which are present in the brains of rats due to different splice variants of the FOLH1 gene $[11,24,25]$ that are not recognized by the antibodies used. Little is known about PSMA isoforms in rats and information about specificity of the used antibodies for different rat PSMA isoforms is not available leaving this question open.

Vascular staining using an antibody against von Willebrand factor showed similar immunofluorescence intensity in the tumor and in the peritumoral area (Fig. 4, suppl. Fig 2), which virtually excludes that differences in neovascularization can explain the prominent tracer binding in the periphery of the tumors. Furthermore, evaluation of microglial activation by CD11b staining demonstrated only a few microglia cells in the peritumoral area. Therefore, it can be assumed that the tracer binding in this area is not caused by microglial activation around the tumor (Fig. 4, suppl. Fig 2).

The examination of the tumor bearing brain slices for activated astrocytes using anti-GFAP staining, however, revealed a strong staining in the peritumoral area of all tumor models (Fig. 4, suppl. Fig 2) which was similar to the pattern of tracer binding at the circumference of the tumors. This observation suggests that the pronounced accumulation of PSMA ligands in the periphery of tumors is associated with reactive astrocytosis. Astrocytes are the most abundant glial cell type in the brain and are involved in many processes such as regulation of brain homeostasis, maintenance and repair of the BBB and neurogenesis [26-28]. Astrocytes are capable of actively reacting to different kinds of neurological disorders by hypertrophy and change of morphology and functions [29]. This process is referred to as reactive astrocytosis and includes the upregulation of GFAP, the main intermediate filament of the astrocytes. In the presence of tumor cells, astrocytes are activated and tend to surround gliomas, exerting both antitumoral and pro-tumoral functions [30]. A high expression of PSMA, also known as glutamate carboxypeptidase II (GCP II), has been reported in astrocytes of the rat brain [31]. Furthermore, investigations of human brain tissue revealed that astrocytes specifically express GCP II in all parts of the human brain [32]. Thus, it seems very likely that the here observed intense accumulation of PSMA ligands in the periphery of all tumor models is caused by binding to reactive astrocytes. The hypothesis is further 
supported by a recent case report, which observed an accumulation of ${ }^{18} \mathrm{~F}$-DCFPyL in a radionecrosis after treatment of a high grade glioma [33]. Since reactive astrocytosis typically occurs in radionecrosis [34], binding of the PSMA ligand to reactive astrocytes would well explain the supposed false positive finding.

The comparison of ${ }^{68} \mathrm{Ga}$-PSMA and ${ }^{18} \mathrm{~F}$-DCFPyL showed differences in uptake at the tumor center and TBR at the tumor margin in all tumor models (Tab. 3). These differences must be interpreted very carefully, since positron range effects of the two different isotopes can have a strong influence, especially in autoradiography [35].

The study is limited by the fact that the specific binding of PSMA ligands to reactive astrocytes could not be documented by corresponding immunostaining using PSMA antibodies. This requires further experiments with different antibodies recognizing specific PSMA isoforms. Furthermore, it is questionable whether the results of the animal experiments are transferable to humans. This is at least supported by the detection of PSMA on human astrocytes as well as the observation of unspecified accumulation of PSMA ligands in a case of radionecrosis as described above.

\section{Conclusions}

The results of this study strongly suggest that reactive astrogliosis can lead to an accumulation of PSMA ligands that significantly exceeds the binding to the actual tumor tissue. This observation could severely limit the validity of the method for assessing brain tumor tissue in patients. In particular this result raises doubts as to whether the method is suitable for theranostics.

\section{Declarations}

\section{Abbreviations}

ANOVA: analysis of variance; AR: autoradiography; BBB: blood-brain barrier; CD11b: antibody against activated microglia; DAPI: 4',6-diamidino-2-phenylindole; Dex: dexamethasone; DMEM: Dulbecco's Modified Eagle's Medium; EBD: Evans blue dye; ${ }^{18}$ F-DCFPyL: 2-(3-(1-carboxy-5-[(6-[ ${ }^{18}$ F]fluoro-pyridine-3carbonyl)-amino]-pentyl)-ureido)-pentanedioic acid; GFAP: glial fibrillary acidic protein; MEM: Minimum Essential Medium Eagle; PMPA: 2-(Phosphonomethyl)pentane-1,5-dioic acid; ${ }^{68} \mathrm{Ga}-\mathrm{PSMA}:{ }^{68} \mathrm{Ga}-\mathrm{Glu}-\mathrm{NH}$ CO-NH-Lys(Ahx)-HBED-CC; PSMA: prostate specific membrane antigen; ROI: regions-of-interest; SUV: standardized uptake value; TBR: tumor-to-background ratio

\section{Ethics approval}

All animals were handled in accordance with the Animal Research Committee of the Forschungszentrum Jülich $\mathrm{GmbH}$, the German Animal Welfare Act and the European Community Council directives regarding the protection of animals used for experimental and scientific purposes (2010/63/EU), and with approval 
by the local ethics committee (Landesamt für Natur, Umwelt und Verbraucherschutz, North-RhineWestphalia, Germany, Az: 84-02.04.2016.248).

\section{Consent for publication}

not applicable

\section{Availability of data and materials}

The datasets used and/or analysed during the current study are available from the corresponding author on reasonable request.

\section{Competing interests}

The authors declare that they have no conflict of interest.

\section{Funding}

No funding was received.

\section{Authors' contributions}

DO, CS, AH, KJL and AW designed the research project and drafted the manuscript, DO and CS performed the experiments under supervision of $\mathrm{AW}, \mathrm{JE}$ and $\mathrm{BN}$ provided radiotracers including quality control and substantially revised the manuscript, NJS and FMM substantially revised the manuscript. All authors read and approved the final manuscript

\section{Acknowledgements}

not applicable

\section{Authors' information}

${ }^{1}$ Institute of Neuroscience and Medicine (INM 4, 5) Forschungszentrum Jülich, Jülich, Germany.

${ }^{2}$ Department of Nuclear Medicine, RWTH Aachen University, Aachen, Germany. ${ }^{3}$ Jülich-Aachen Research Alliance (JARA)-Section JARA-Brain, Aachen, Germany.

${ }^{4}$ Department of Neurology, RWTH Aachen University, Aachen, Germany, ${ }^{5}$ Department of Radiology and Nuclear Medicine, Maastricht University Medical Center (MUMC+), Maastricht, The Netherlands 


\section{References}

1.Ekmekcioglu O, Busstra M, Klass ND, Verzijlbergen F. Bridging the Imaging Gap: PSMA PET/CT Has a High Impact on Treatment Planning in Prostate Cancer Patients with Biochemical Recurrence-A Narrative Review of the Literature. J Nucl Med. 2019;60:1394-8.

2.Tan N, Bavadian N, Calais J, Oyoyo U, Kim J, Turkbey IB, et al. Imaging of Prostate Specific Membrane Antigen Targeted Radiotracers for the Detection of Prostate Cancer Biochemical Recurrence after Definitive Therapy: A Systematic Review and Meta-Analysis. J Urol. 2019;202:231-40.

3.Szabo Z, Mena E, Rowe SP, Plyku D, Nidal R, Eisenberger MA, et al. Initial Evaluation of [(18)F]DCFPyL for Prostate-Specific Membrane Antigen (PSMA)-Targeted PET Imaging of Prostate Cancer. Mol Imaging Biol. 2015;17:565-74.

4.Dietlein F, Kobe C, Neubauer S, Schmidt M, Stockter S, Fischer T, et al. PSA-Stratified Performance of (18)F- and (68)Ga-PSMA PET in Patients with Biochemical Recurrence of Prostate Cancer. J Nucl Med. 2017;58:947-52.

5.Chang SS, Reuter VE, Heston WD, Bander NH, Grauer LS, Gaudin PB. Five different anti-prostate-specific membrane antigen (PSMA) antibodies confirm PSMA expression in tumor-associated neovasculature. Cancer Res. 1999;59:3192-8.

6.Salas Fragomeni RA, Amir T, Sheikhbahaei S, Harvey SC, Javadi MS, Solnes LB, et al. Imaging of Nonprostate Cancers Using PSMA-Targeted Radiotracers: Rationale, Current State of the Field, and a Call to Arms. J Nucl Med. 2018;59:871-7.

7.Morgenroth A, Tinkir E, Vogg ATJ, Sankaranarayanan RA, Baazaoui F, Mottaghy FM. Targeting of prostate-specific membrane antigen for radio-ligand therapy of triple-negative breast cancer. Breast Cancer Res. 2019;21:116.

8.Endepols H, Morgenroth A, Zlatopolskiy BD, Krapf P, Zischler J, Richarz R, et al. Peripheral ganglia in healthy rats as target structures for the evaluation of PSMA imaging agents. BMC Cancer. 2019;19:633.

9.Bailly C, Vidal A, Bonnemaire C, Kraeber-Bodere F, Cherel M, Pallardy A, et al. Potential for Nuclear Medicine Therapy for Glioblastoma Treatment. Front Pharmacol. 2019;10:772.

10.Wernicke AG, Edgar MA, Lavi E, Liu H, Salerno P, Bander NH, et al. Prostate-specific membrane antigen as a potential novel vascular target for treatment of glioblastoma multiforme. Arch Pathol Lab Med. 2011;135:1486-9.

11.Nomura N, Pastorino S, Jiang P, Lambert G, Crawford JR, Gymnopoulos M, et al. Prostate specific membrane antigen (PSMA) expression in primary gliomas and breast cancer brain metastases. Cancer Cell Int. 2014;14:26. 
12.Schwenck J, Tabatabai G, Skardelly M, Reischl G, Beschorner R, Pichler B, et al. In vivo visualization of prostate-specific membrane antigen in glioblastoma. Eur J Nucl Med Mol Imaging. 2015;42:170-1.

13.Sasikumar A, Joy A, Pillai MR, Nanabala R, Anees KM, Jayaprakash PG, et al. Diagnostic Value of 68Ga PSMA-11 PET/CT Imaging of Brain Tumors-Preliminary Analysis. Clin Nucl Med. 2017;42:e41-e8.

14.Unterrainer M, Niyazi M, Ruf V, Bartenstein P, Albert NL. The endothelial prostate-specific membrane antigen is highly expressed in gliosarcoma and visualized by [68Ga]-PSMA-11 PET: a theranostic outlook for brain tumor patients? Neuro Oncol. 2017;19:1698-9.

15.Salas Fragomeni RA, Menke JR, Holdhoff M, Ferrigno C, Laterra JJ, Solnes LB, et al. Prostate-Specific Membrane Antigen-Targeted Imaging With [18F]DCFPyL in High-Grade Gliomas. Clin Nucl Med. 2017;42:e433-e5.

16.Langen KJ, Jarosch M, Muhlensiepen H, Hamacher K, Broer S, Jansen P, et al. Comparison of fluorotyrosines and methionine uptake in F98 rat gliomas. Nucl Med Biol. 2003;30:501-8.

17.Dietlein M, Kobe C, Kuhnert G, Stockter S, Fischer T, Schomacker K, et al. Comparison of [(18)F]DCFPyL and [ (68)Ga]Ga-PSMA-HBED-CC for PSMA-PET Imaging in Patients with Relapsed Prostate Cancer. Mol Imaging Biol. 2015;17:575-84.

18.Geisler S, Willuweit A, Schroeter M, Zilles K, Hamacher K, Galldiks N, et al. Detection of remote neuronal reactions in the Thalamus and Hippocampus induced by rat glioma using the PET tracer cis-4[(1)(8)F]fluoro-D-proline. J Cereb Blood Flow Metab. 2013;33:724-31.

19.Endepols H, Mottaghy FM, Simsekyilmaz S, Bucerius J, Vogt F, Winz O, et al. In vivo Molecular Imaging of Glutamate Carboxypeptidase II Expression in Re-endothelialisation after Percutaneous Balloon Denudation in a Rat Model. Sci Rep. 2018;8:7411.

20.Silver DA, Pellicer I, Fair WR, Heston WD, Cordon-Cardo C. Prostate-specific membrane antigen expression in normal and malignant human tissues. Clin Cancer Res. 1997;3:81-5.

21.Jackson PF, Cole DC, Slusher BS, Stetz SL, Ross LE, Donzanti BA, et al. Design, synthesis, and biological activity of a potent inhibitor of the neuropeptidase $\mathrm{N}$-acetylated alpha-linked acidic dipeptidase. J Med Chem. 1996;39:619-22.

22.Slusher BS, Vornov JJ, Thomas AG, Hurn PD, Harukuni I, Bhardwaj A, et al. Selective inhibition of NAALADase, which converts NAAG to glutamate, reduces ischemic brain injury. Nat Med. 1999;5:1396402.

23.Stegmayr C, Bandelow U, Oliveira D, Lohmann P, Willuweit A, Filss C, et al. Influence of blood-brain barrier permeability on O-(2-(18)F-fluoroethyl)-L-tyrosine uptake in rat gliomas. Eur J Nucl Med Mol Imaging. 2017;44:408-16. 
24.Schmittgen TD, Teske S, Vessella RL, True LD, Zakrajsek BA. Expression of prostate specific membrane antigen and three alternatively spliced variants of PSMA in prostate cancer patients. Int $\mathrm{J}$ Cancer. 2003;107:323-9.

25.Cao KY, Xu L, Zhang DM, Zhang XM, Zhang T, He X, et al. New alternatively spliced variant of prostatespecific membrane antigen PSM-E suppresses the proliferation, migration and invasiveness of prostate cancer cells. Int J Oncol. 2012;40:1977-85.

26.Volterra A, Meldolesi J. Astrocytes, from brain glue to communication elements: the revolution continues. Nat Rev Neurosci. 2005;6:626-40.

27.Abbott NJ, Ronnback L, Hansson E. Astrocyte-endothelial interactions at the blood-brain barrier. Nat Rev Neurosci. 2006;7:41-53.

28.Quinones-Hinojosa A, Chaichana K. The human subventricular zone: a source of new cells and a potential source of brain tumors. Exp Neurol. 2007;205:313-24.

29. Hol EM, Pekny M. Glial fibrillary acidic protein (GFAP) and the astrocyte intermediate filament system in diseases of the central nervous system. Curr Opin Cell Biol. 2015;32:121-30.

30.0'Brien ER, Howarth C, Sibson NR. The role of astrocytes in CNS tumors: pre-clinical models and novel imaging approaches. Front Cell Neurosci. 2013;7:40.

31. Berger UV, Luthi-Carter R, Passani LA, Elkabes S, Black I, Konradi C, et al. Glutamate carboxypeptidase II is expressed by astrocytes in the adult rat nervous system. J Comp Neurol. 1999;415:52-64.

32.Sacha P, Zamecnik J, Barinka C, Hlouchova K, Vicha A, Mlcochova P, et al. Expression of glutamate carboxypeptidase II in human brain. Neuroscience. 2007;144:1361-72.

33.Salas Fragomeni RA, Pienta KJ, Pomper MG, Gorin MA, Rowe SP. Uptake of Prostate-Specific Membrane Antigen-Targeted 18F-DCFPyL in Cerebral Radionecrosis: Implications for Diagnostic Imaging of High-Grade Gliomas. Clin Nucl Med. 2018;43:e419-e21.

34.Miyatake S, Nonoguchi N, Furuse M, Yoritsune E, Miyata T, Kawabata S, et al. Pathophysiology, Diagnosis, and Treatment of Radiation Necrosis in the Brain. Neurol Med Chir (Tokyo). 2015;55 Suppl $1: 50-9$.

35.Alva-Sanchez H, Quintana-Bautista C, Martinez-Davalos A, Avila-Rodriguez MA, Rodriguez-Villafuerte M. Positron range in tissue-equivalent materials: experimental microPET studies. Phys Med Biol. 2016;61:6307-21.

\section{Tables}


Due to technical limitations, Tables 1 - 4 are only available for download from the Supplementary Files section.

\section{Figures}
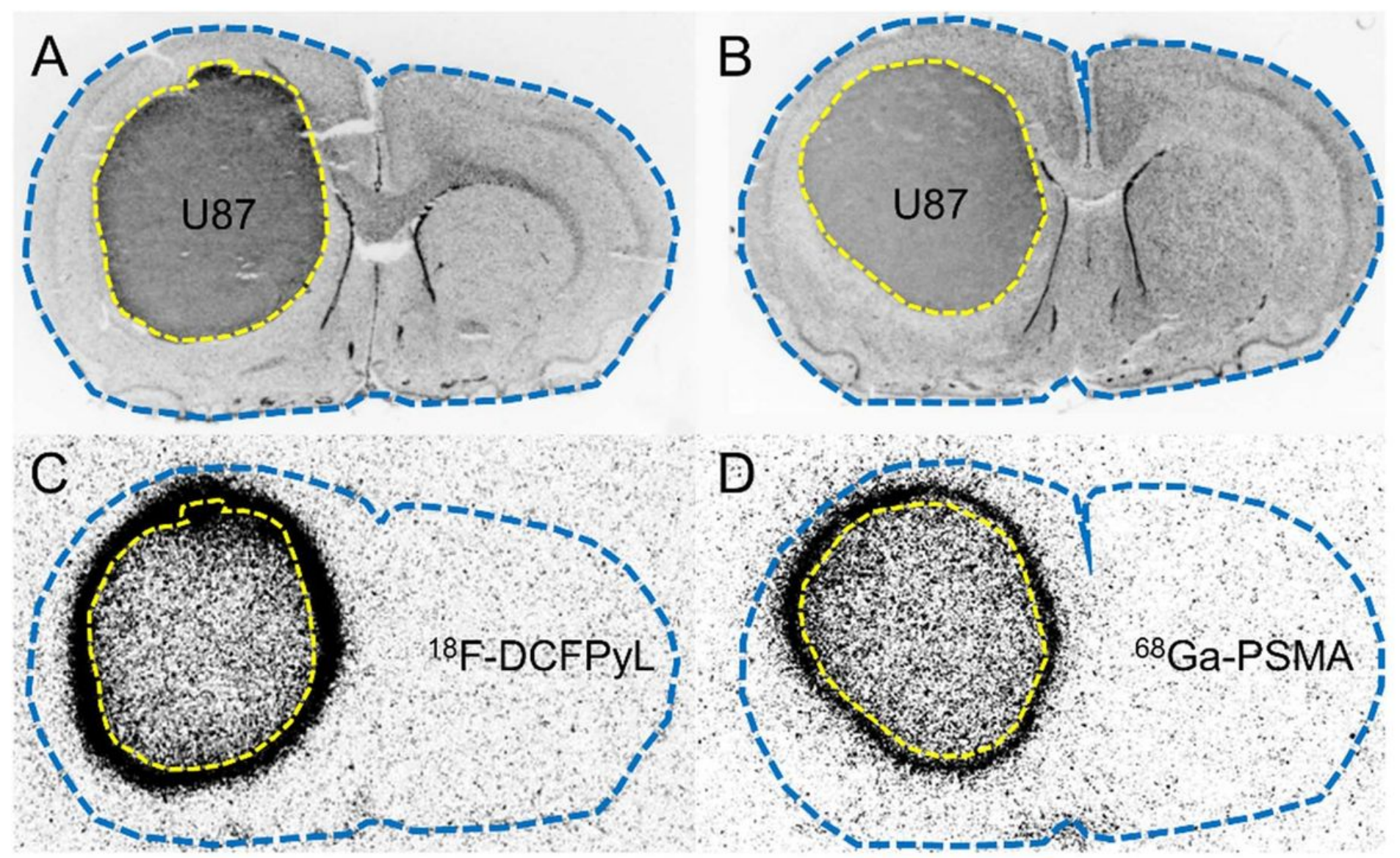

Figure 1

Comparison of histological stainings (nuclear staining with DAPI, A, B) and autoradiograms (C, D) of U87 rat gliomas. Tumor tissue is outlined by a dotted yellow line in the histological slices $(A, B)$ and projected onto the corresponding autoradiograms $(C, D)$. The outer edge of brain tissue is outlined by a dotted blue line. Both the 18F-DCFPyL (C) and 68Ga-PSMA autoradiograms (D) demonstrate prominent uptake in the peritumoral region. 

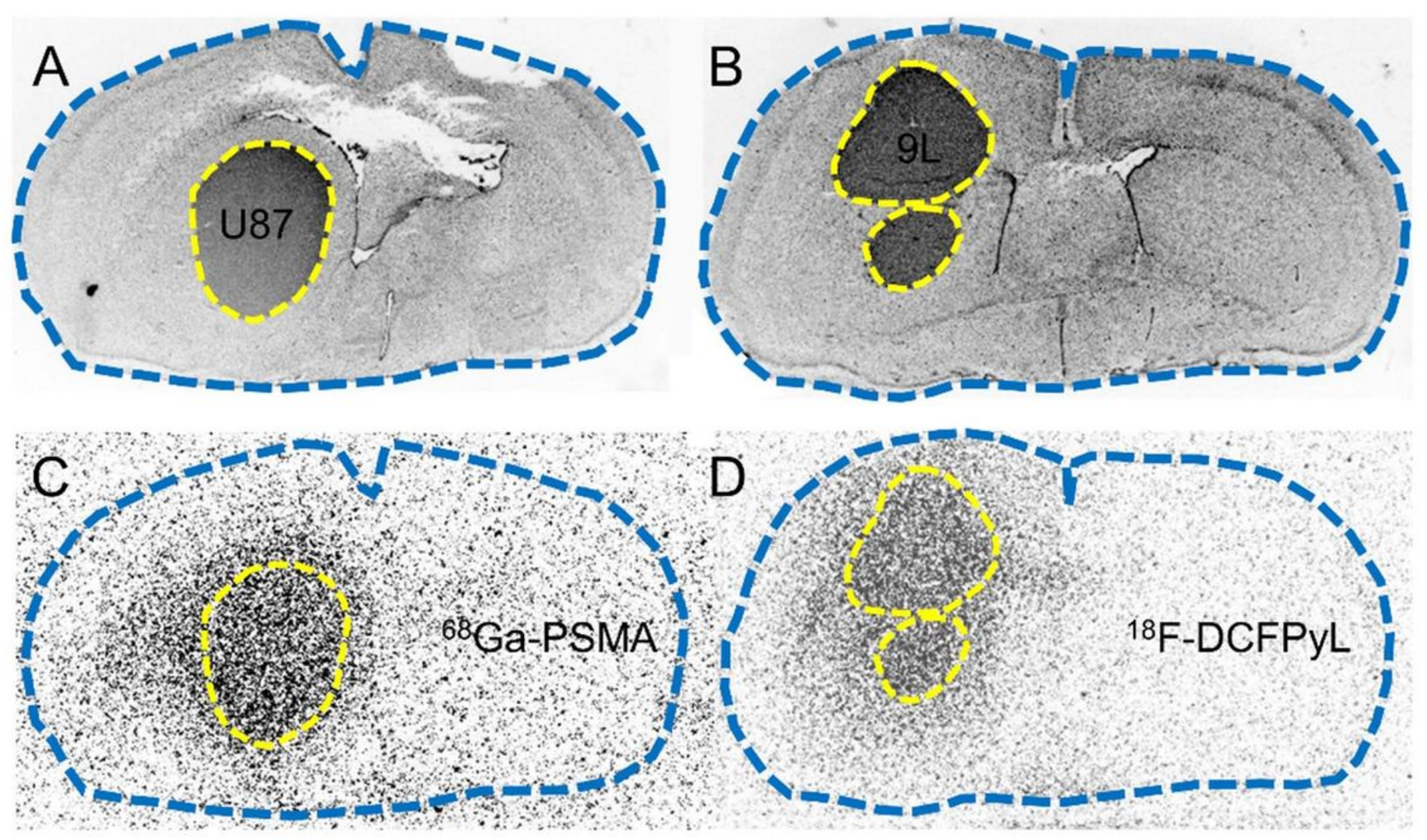

Figure 2

Influence of PMPA competition on 68Ga-PSMA and 18F-DCFPyL uptake in U87 (left) and 9L glioma (right), respectively. Coronal brain slices of histological staining (A, B) and autoradiography (C, D). Tumor tissue is outlined as a dotted yellow line in the nuclear staining and projected onto the autoradiograms, and the outer edge of brain tissue is outlined by a blue dotted line. Co-injection of PMPA blocks 68GaPSMA uptake in the periphery of the $9 \mathrm{~L}$ glioma (C) and 18F-DCFPyL uptake in U87 glioma (D). 

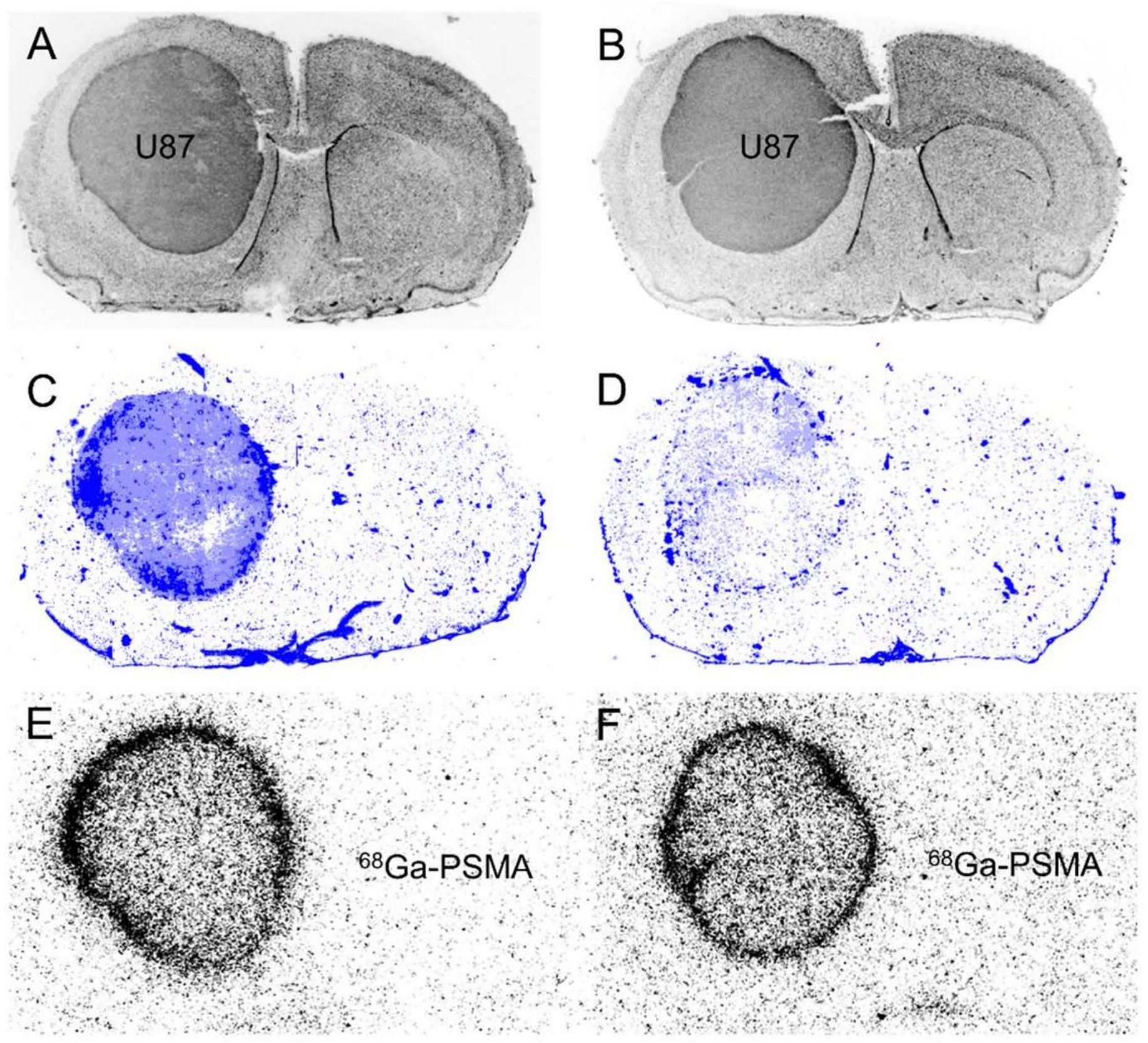

\section{Figure 3}

Coronal brain slices of an untreated (left column) and dexamethasone (Dex) treated U87 bearing rat (right column). Histological staining (DAPI) (A, B), Evans blue staining (C, D) and 68Ga-PSMA autoradiography $(E, F)$. The untreated animal shows a strong extravasation of Evans blue into the tumor tissue indicating BBB disruption (C). In contrast, the Dex treated animal shows little extravasation of Evans blue into tumor tissue indicating a restoration of the BBB after Dex treatment (D). 68Ga-PSMA autoradiography shows an unchanged prominent spherical tracer binding at the tumor rim both in the untreated animal (E) and after Dex treatment $(F)$. 


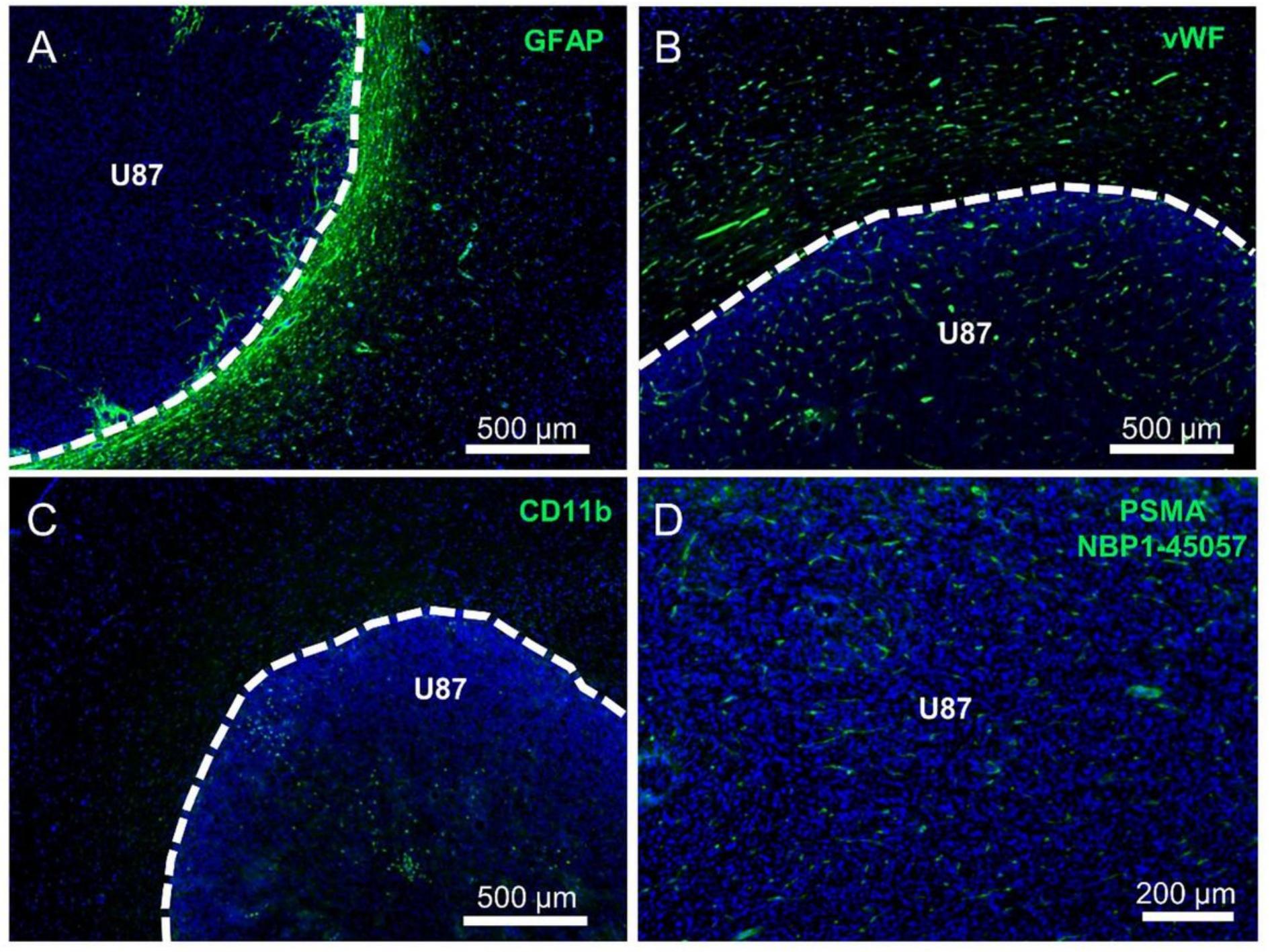

Figure 4

Immunofluorescence staining of U87 tumors. Nuclear staining (DAPI) is shown in blue, antibody staining in green, and tumor borders are outlined in white. Strong reactive astrocytosis (GFAP; A) is visible in the peritumoral region with few astrocytes at the tumor border. Specific staining of blood vessels (vWF; B) is detectable, which is similar within tumor tissue and in the peritumoral region. Few activated microglia (CD11b; C) can be observed in the peritumoral region and inside the tumor. PSMA staining using the antibody NBP1-45057 is positive in vessel-like structures in the tumor center (D) but not outside the tumor.

\section{Supplementary Files}

This is a list of supplementary files associated with this preprint. Click to download.

- tables.docx

- SupplementalMaterialfinal.DOCX 